\title{
Influence of Childhood Trauma and Personality Traits on Paranormal Beliefs among Early-adults
}

\author{
Aswathi Prasad ${ }^{1}$, Baiju Gopal ${ }^{2}$
}

\section{ABSTRACT}

The field of Parapsychology is concerned with the exploration of psychic and paranormal phenomena which has deep-rooted repercussion in the social world from time immemorial. Research in this field has gone beyond bounds and leaps in distant cultural contexts, yet the local milieu stands right in the middle of the road. The present study which intended to find out the influence of childhood trauma and personality beliefs on paranormal beliefs was conducted among early adults belonging to the age group 18-30 years. The sample consisted of 190 early-adults (67 males and 123 females) hailing from different districts of Kerala, India. Purposive sampling method was employed for the selection of participants. The tools used for the study include Revised Paranormal Beliefs Scale (Tobacyk, 1983), Childhood trauma Questionnaire (Pennebacker and Susman, 2013), and Big Five-Factor Inventory (44 items). It was found that there are significant relationships between few dimensions of paranormal beliefs, childhood trauma, and personality traits as proposed earlier, and certain dimensions of childhood trauma and personality traits are significant predictors of paranormal beliefs. The study also found that the distribution of different dimensions of paranormal beliefs is not significant across categories of gender (males and females), except in the case of one dimension. An exploration into the backdrop of paranormal beliefs among individuals hailing from the venerable culture of Kerala; anchored in heritage and religiosity, might be of assistance in this regard. This study could probably be reflected as such an endeavour to unfurl mysteries hidden in the regional psyche.

\section{Keywords: Paranormal beliefs, Childhood trauma, Personality traits, Coping mechanism}

The expeditions of modern science and technology have been escalating in every millisecond by all means. A glimpse into the advancement of modern science insists a spectator to marvel at the elegance of innovations and the profundity of pragmatism. The field of Parapsychology is concerned with the exploration of psychic and paranormal phenomena which has deeprooted repercussion in the social world from time immemorial. The existence of paranormal beliefs in the minds of individuals is evident in their behavior, response patterns as well as

\footnotetext{
${ }^{1}$ M.Sc Psychology (Clinical), Christ University, Bengaluru, India

${ }^{2}$ Associate Professor, Department of Psychology, Christ University, India

*Responding Author

(C) 2017 Prasad A \& Gopal B; licensee IJIP. This is an Open Access Research distributed under the terms of the Creative Commons Attribution License (www.creativecommons.org/licenses/by/2.0), which permits unrestricted use, distribution, and reproduction in any Medium, provided the original work is properly cited.
} 
daily life routines. Research in this field has gone beyond bounds and leaps in distant cultural contexts, yet the local milieu stands right in the middle of the road. Pasricha (2011) wrote that the subject matter of parapsychology, that is the paranormal phenomena; transcend the boundaries of time, space and force. Interestingly, the art of knowing the unknown collaborates with multiple layers of the psyche in various aspects. A spectator would come across a multitude of such concepts while probing into the subject matter of parapsychology, ranging from a sense of acceptance towards beliefs in the anomaly till the extra ordinary human potentials to control the order of nature. Lindenan and Aarnio (2006) defined Paranormal beliefs as the "beliefs in physical, biological or psychological phenomena that feature core ontological properties of another anthological category". According to them, the whole picture of paranormal beliefs has been obscure due to the presence of a host of cognitive, affective, social and motivational correlates of paranormal beliefs, having no proper known antecedents (Lindeman \& Aarrnio, 2006). Paranormal beliefs entail fervor to the existence of strokes pertaining to ghosts, extra sensory perceptual beliefs or psychic abilities, extra terrestrial life, near-death experiences, out-of-body experiences, un-identified flying objects or cryptids. According to Tobacyk (1983), there are seven major dimensions of paranormal beliefs such as Traditional religious beliefs, Psychology Slander Intuition (Psi), Witchcraft, Superstition, Spiritualism, Extra-ordinary life forms, and Pre-cognition. Beliefs pertaining to these domains are considered to have several supporting factors ranging from genetic elements to socio-cultural settings.

While considering the plausible developmental attributes, researches have shown that the incidence of abusive and traumatic events in the childhood could be positively correlated with the beliefs in the paranormal Phenomena (as suggested by Irwin, 1992, 1994 ; Lawrence, Edwards, Barraclough, Church \& Hetherington, 1995). However, Irwin (2005) pointed out that the belief in the paranormal phenomena may originate due to the need for coping up perceived lack of control resulting from any relatively traumatic events in childhood and certain other common forms of childhood experiences associated with a lack of command over own life, such as having authoritarian parents or being a younger sibling. So these studies suppose that individuals may rely on paranormal beliefs as a coping mechanism to cope up the impact of a childhood traumatic experience in later life.

Thalbourne (1995) argued that individual differences associated with paranormal beliefs should be further investigated and the existing results or inferences should be verified in heterogeneous populations (as cited by Karl Peltzer in 2002). Karl Pettzer (2002) later hypothesised that the research in the field of paranormal beliefs can provide greater insight into the nature of the paranormal phenomena, the nature of people who rely on such phenomena, and the concurrent experiences that resulted in the uprooting of such paranormal beliefs. It was also agreed that the personality factors and locus of control can be linked with an enhanced level of belief in paranormal phenomena. Since personality can be considered as the set of psychological traits and mechanisms in the individual that are organized and relatively enduring and that influence bigger interactions with and adaptations to the environment, it is possible to put forward an assumption that paranormal beliefs are rightly 
linked with the personality traits, as such enduring patterns of individual differences exert a constant influence on a person's belief systems, behavior and life. Over the last few decades, several researchers have looked at the association between these two factors in different contexts. Paranormal beliefs were analysed by rubbing on different dimensions of personality including openness to experience, extraversion agreeableness, neuroticism and conscientiousness. This possible correlation is, however, can be an eye opener to the wellknit association between the psyche and the dimensions of personality. Several researchers have reported the Big-Five Factors of personality as having a predominant linkage with the paranormal, superstitious and extraterrestrial beliefs. Some studies have suggested that paranormal beliefs and personality dimensions of neuroticism and extraversion are entangled with each other. Other dimensions including agreeableness, openness to experiences and conscientiousness are also considered to be the determinants of belief patterns and systems. Another major study also pointed out that it is possible to presume the extent of paranormal beliefs held by a person by examining an array of personality traits and other individual differences (Swami, Pietcshing, Stieger \& Voracek, 2011).

The study presumed that paranormal beliefs act as a psychodynamic coping mechanism and individuals tend to seize such beliefs to deal with traumatic experiences of life, especially childhood trauma (prior to age 17). Individual predispositions and personality traits have also been accounted to exert a significant influence with the possession of belief systems. In fact it is surprising to know that early adults of this scientific era hold on to a couple of paranormal beliefs, other than traditional religious principles, due to unfathomable reasons of their own. The indispensable rationale behind this study was the urge to probe into such a fascinating fact from a psychological perspective. Since early adulthood is a crucial face in which the individual predominantly reconcile his belief systems that play a vital role in shaping the life structure, an understanding about the origins of such beliefs could assist in providing appropriate psychological intrusions, if necessary.

\section{Objectives of the Study}

\section{Major Objective}

- $\quad$ To find out the influence of childhood trauma and personality beliefs on paranormal beliefs among early adults.

\section{Specific Objectives}

- $\quad$ To understand whether there exist any correlation between paranormal beliefs and personality traits.

- $\quad$ To understand whether there is a significant relationship between the experience of childhood trauma and paranormal beliefs.

- To understand the gender differences in the possession of paranormal beliefs precipitated by the experience of childhood trauma and personality traits.

\section{Hypotheses}

Hypothesis 1: There is a difference in the distribution of paranormal beliefs between males and females (across categories of gender). 
Specific Hypotheses

H1a: There is a difference in the distribution of Traditional religious beliefs across categories of gender

H1b: There is a difference in the distribution of beliefs in Psi across categories of gender.

H1c: There is a difference in the distribution of Witchcraft across categories of gender.

H1d: There is a difference in the distribution of beliefs in Superstition across categories of gender.

H1e: There is a difference in the distribution of beliefs in Spiritualism across categories of gender.

H1f: There is a difference in the distribution of beliefs in Extraordinary life forms across categories of gender.

H1g: There is a difference in the distribution of beliefs in Pre-Cognition across categories of gender

Hypothesis 2: There exists a relationship between Paranormal beliefs and Childhood trauma. Hypothesis 3: There exists a relationship between Paranormal beliefs and Personality traits.

\section{METHOD}

\section{Sample}

The study consisted of 190 young adults - 67 males and 123 females belonging to the age group 18-30 years. The participants were selected across all the districts in Kerala, India. Purposive Sampling was used to identify the sample population. The researcher had approached potential participants aiming at getting representation from various parts of the state.

\section{Research Design}

The study adopted a correlational strategy where the relationship between Childhood Trauma, Personality Traits, and Paranormal Beliefs are explored in a purposive manner.

\section{Instruments}

1. Revised Paranormal Belief Scale (RPBS). This scale which includes 26-items that provide a measure of the degree of beliefs in paranormal phenomena was developed by Jerome J. Tobacyk. It is a seven-point rating Scale. There are seven dimensions such as Traditional religious belief, psi, witchcraft, superstition, spiritualism, extra-ordinary life forms, and Pre-cognition.

2. Childhood trauma questionnaire (CTQ). This scale that measures the degree to which an individual has experienced traumatic events in the childhood was developed by Pennebaker and Susman (2013). It deals with 6 items that screens any trauma that was experienced by the individual prior to the age of 17 .

3. Big-five Factor Inventory (BFI). It is a 44- item inventory that measures the degree of big five factor dimensions present in an individual's personality. The big-five factors are extraversion Vs. Introversion, agreeableness Vs antagonism, conscientiousness vs. lack 
Influence of Childhood Trauma and Personality Traits on Paranormal Beliefs among Early-adults

of detection, Neuroticism vs. emotional stability, and openness vs. closeness to experience.

\section{Data analysis}

The data analysis was conducted with the help of SPSS software (Version 23) and Microsoft excel. Normality analysis (Shapiro-Wilk test), Mann-Whitney U test, Spearman's Rank order correlation, and Stepwise Regression analysis were the statistical operations performed.

\section{Ethical considerations}

Informed consent was taken prior to the study. Strict confidentiality has been maintained throughout the research. No Physical or mental harm was done to the participants of the study at any point in time. The participants were ensured with all rights to withdraw at any time during the study. They were also intimated of any potential risks or discomforts involved in participation beforehand.

\section{RESULTS}

Table 1, Sample description

\begin{tabular}{|l|l|l|l|l|l|}
\hline Gender & $\begin{array}{l}\text { Age } \\
\text { range }\end{array}$ & $\begin{array}{l}\text { Mean } \\
\text { age }\end{array}$ & Frequency & Percent & $\begin{array}{l}\text { Cumulative } \\
\text { percent }\end{array}$ \\
\hline Male & $18-30$ & 22.52 & 123 & 64.7 & 64.7 \\
\hline Female & (Years) & 20.98 & 67 & 35.3 & 100 \\
\hline Total & & & 190 & 100 & \\
\hline
\end{tabular}

As shown in Table 1, the total sample size $(N)=190$, out of which 123 were females and 67 were males. This indicates that the sample distribution accounted for $64.7 \%$ of females and $35.3 \%$ of males (in terms of gender). The participants belonged to the age group $18-30$ years. The mean age of males was found to be 22.52 years and the mean age of females was 20.98 years.

Table 2, Descriptive Statistics showing Mean and SD of Paranormal Beliefs

\begin{tabular}{|l|l|l|l|}
\hline Variable (Dimension) & N & Mean & SD \\
\hline $\begin{array}{l}\text { Traditional Religious } \\
\text { Beliefs }\end{array}$ & 190 & 20.458 & 5.7538 \\
\hline Psi & 190 & 13.153 & 5.7018 \\
\hline Witchcraft & 190 & 12.663 & 6.3331 \\
\hline Superstition & 190 & 5.953 & 3.7307 \\
\hline Spiritualism life & 190 & 13.458 & 6.0967 \\
\hline $\begin{array}{l}\text { Extra-Ordinary } \\
\text { forms }\end{array}$ & 9.358 & 3.6772 \\
\hline Pre-Cognition & 190 & 12.821 & 6.2859 \\
\hline
\end{tabular}

Table 2 demonstrates the descriptive statistics of paranormal beliefs across the sample distribution. It is implied that Traditional religious beliefs has the highest mean score $(M=20.45)$ and Superstition has the lowest mean score $(M=5.95)$. Witchcraft has the highest SD score $(S D=6.33)$ and Extra-ordinary life forms has the lowest SD score $(S D=3.67)$.

(C) The International Journal of Indian Psychology, ISSN 2348-5396 (e) | ISSN: 2349-3429 (p) | 135 
Hypothesis 1: There is a difference in the distribution of paranormal beliefs between males and females (across categories of gender).

It was found that there is no significant difference in the distribution of paranormal beliefs across categories of Gender, except in the case of beliefs in Witchcraft ( $U=1242.500, z=-$ 2.938, $p=.003)$. Hence, only Hypothesis -1c (H1c) is accepted.

Table 3, Mann-Whitney U test of Witchcraft ( $3^{\text {rd }}$ dimension of Paranormal beliefs)

\begin{tabular}{|c|c|c|c|c|c|c|}
\hline $\begin{array}{l}\text { Variable } \\
\text { (Dimension) }\end{array}$ & Group & $\mathbf{N}$ & $\begin{array}{l}\text { Mean } \\
\text { Rank }\end{array}$ & $\mathbf{U}$ & $Z$ & Sig. \\
\hline \multirow{3}{*}{ Witchcraft } & Males & 60 & 51.21 & \multirow{3}{*}{1242.500} & \multirow{3}{*}{-2.938} & \multirow{3}{*}{.003} \\
\hline & Females & 60 & 69.79 & & & \\
\hline & Total & 120 & & & & \\
\hline
\end{tabular}

$* * p<.05$

Table 3 indicates that there is a significant difference in the distribution of beliefs in Witchcraft across categories of Gender $(U=1242.500, z=-2.938, p=.003)$. It is evident that females were found to have more significant beliefs in witchcraft than males (females have higher mean rank score than males). Tobacyk and Milford (1983) have opined that females tend to have higher measures global paranormal beliefs when compared to men. Since Witchcraft is one of the prominent realms of global paranormal beliefs, it could be presumed that the results of the present study have been corresponding to previous researches. Huntley (2013) also found that there is a significant difference among males and females with regard to their beliefs in Witchcraft.

Hypothesis 2: There exists a relationship between Paranormal beliefs and Childhood trauma.

Table 4, Spearman's Rank order correlation coefficient matrix of Paranormal beliefs and Childhood trauma

\begin{tabular}{|l|l|l|l|l|l|l|}
\hline $\begin{array}{l}\text { Variables } \\
\text { (Dimensions) }\end{array}$ & Death & $\begin{array}{l}\text { Divorce or } \\
\text { Separation }\end{array}$ & $\begin{array}{l}\text { Sexual } \\
\text { Abuse }\end{array}$ & $\begin{array}{l}\text { Violence } \\
\text { Victimization }\end{array}$ & $\begin{array}{l}\text { Physical } \\
\text { Injury or } \\
\text { Illness }\end{array}$ & $\begin{array}{l}\text { Significant } \\
\text { Life } \\
\text { Experiences }\end{array}$ \\
\hline $\begin{array}{l}\text { Traditional } \\
\text { Religious } \\
\text { Beliefs }\end{array}$ & .091 & .005 & .016 & -.066 & .085 & .040 \\
\hline Psi & $.166^{*}$ & -.019 & .122 & .087 & $.167 *$ & $.172^{*}$ \\
\hline Witchcraft & $.218^{* *}$ & -.009 & .069 & .004 & .106 & .100 \\
\hline Superstition & $.276^{* *}$ & 0.27 & -.017 & -.061 & .009 & -.030 \\
\hline Spiritualism & $.208^{* *}$ & .035 & .066 & .022 & .100 & .115 \\
\hline $\begin{array}{l}\text { Extra } \\
\text { Ordinary Life } \\
\text { forms }\end{array}$ & $.217^{* *}$ & .014 & -.012 & .049 & .080 & $.145^{*}$ \\
\hline $\begin{array}{l}\text { Pre- } \\
\text { Cognition }\end{array}$ & $.174^{*}$ & -.017 & .017 & -.118 & -.027 & .056 \\
\hline
\end{tabular}

$* p<.05$ (2-tailed).

$* * p<.01$ (2-tailed).

(C) The International Journal of Indian Psychology, ISSN 2348-5396 (e)| ISSN: 2349-3429 (p) | 136 
Table 4 shows Spearman's rank order correlation analysis between different dimensions of Paranormal beliefs and Childhood trauma. Irwin (1994), who suggested that people who deeply rely on paranormal phenomena tend to have a childhood history of traumatic experiences, also found that a sample of 32 adults whose parents were alcoholics (adults who suffered due to substance abuse and dependence among their parents in childhood) had stronger beliefs in Witchcraft, Superstition, and Pre-cognition; when compared to a control group constituted by 89 participants. Results of the present study could also be aligned with respect to this observation. Spearman's rank order correlation indicates that there is no significant correlation between Traditional religious beliefs and any other dimension of Childhood trauma. It was fond that beliefs in Psi has significant positive correlations with childhood traumatic experiences of Death ( $r s=.166, p=.022)$, Physical illness or injury ( $r s=$ $.167, p=.021)$, and Significant life experiences ( $r s=.172, p=.017)$. But there is no significant correlation between other dimensions of childhood trauma and the beliefs in Psi. Spearman's rank order correlation analysis has shown that there is a positive correlation between Witchcraft and Death $(r s=.218, p=.003)$, thereby indicating that there is significant relationship between the two variables. But, no significant correlation could be found between beliefs in Witchcraft and other dimensions of Childhood traumatic experiences. The results of Spearman's correlation analysis revealed that Death was again a significant correlate of the beliefs in Superstition ( $r s=.276, p=.000$ ), Spiritualism $(r s=.208, p=.004$ ), Extra-ordinary life forms ( $r s=.217, p=.003$ ), and Pre-cognition ( $r s=.174, p=.016)$. It is also shown that there exists a positive correlation between Significant life experiences and the beliefs in Extra-ordinary life forms ( $r s=.145, p=.046$ ), thereby indicating that there is a significant relationship between the two variables. But no significant correlations were found between the other dimensions of Childhood trauma and Paranormal beliefs. Hence, Hypothesis -2 could be accepted in the case of these 9 combinations of paranormal beliefs and childhood traumatic experiences (where significant correlation could be found), and subjected to rejection otherwise.

Hypothesis 3: There exists a relationship between Paranormal beliefs and Personality traits.

Table 5, Spearman's rank order correlation coefficient matrix of Paranormal beliefs and Personality traits

\begin{tabular}{|c|c|c|c|c|c|}
\hline $\begin{array}{l}\text { Variables } \\
\text { (Dimensions) }\end{array}$ & Extraversion & Agreeableness & conscientiousness & Neuroticism & $\begin{array}{l}\text { Openness } \\
\text { to } \\
\text { Experience }\end{array}$ \\
\hline $\begin{array}{l}\text { Traditional } \\
\text { Religious } \\
\text { Beliefs }\end{array}$ & .075 & .117 & $.176^{*}$ & .003 & -.003 \\
\hline Psi & -.045 & -.094 & -.112 & .130 & .131 \\
\hline Witchcraft & -.136 & -.075 & -.056 & $.189^{* *}$ & .127 \\
\hline Superstition & $-.181 *$ & $-.162^{*}$ & -.048 & $.150 *$ & .108 \\
\hline $\begin{array}{l}\text { Spiritualism } \\
\text { Extra- }\end{array}$ & .083 & -.103 & .133 & .020 & $.201^{* *}$ \\
\hline $\begin{array}{l}\text { Ordinary Life } \\
\text { forms }\end{array}$ & .010 & -.025 & .085 & -.045 & $.171^{*}$ \\
\hline Pre- Cognition & -.135 & .040 & .018 & .057 & .094 \\
\hline
\end{tabular}

$* p<.05$ (2-tailed).

$* * p<.01$ (2-tailed). 
Table 5 demonstrates Spearman's rank order correlation analysis of Personality traits and the beliefs in Paranormal beliefs. It was found that there exists a positive correlation between Traditional religious beliefs and Conscientiousness ( $r s=.176, p=.015$ ), thereby indicating a significant relationship between the two variables. The results indicate that the beliefs in Psi were not significantly correlated to any of the Personality traits. The beliefs in Witchcraft was shown to have a significant relationship with Neuroticism ( $r s=.189, p=.009$ ). Similarly, the beliefs in Superstition was also found to have a significant positive correlation with Neuroticism ( $r s=.150, p=.039$ ). As suggested by previous studies mentioned earlier, it was revealed that the trait of Neuroticism has a positive association with paranormal beliefs. Huntley (2013) have found that neuroticism could not be correlated with any dimension of paranormal beliefs, other than Superstition. However, this proposition should be explored further. Moving on, the analysis revealed that beliefs in Superstition has a significant negative correlation with Extraversion ( $r s=-.181, p=.013$ ), and Agreeableness ( $r s=-.162$, $p=.025$ ). Huntley (2013) opined that the correlation between Agreeableness with paranormal beliefs, especially with domains directly linked to religiosity, could probably be considered as a partial evidence for the findings of previous studies claiming low psychoticism and religiosity stemming out of agreeableness. It was also noted that there is a positive relationship between Extraversion and paranormal beliefs that could be explained by the nature of extraverted persons, especially their extreme receptive attitudes. However, the results of the present study do not support that proposition. Results demonstrate that Spiritualism has a positive correlation with Openness to Experience ( $r s=.201, p=.005$ ), thereby indicating a significant relationship between these two variables. The beliefs in Extra-ordinary life forms was also found to have a significant positive correlation with Openness to Experience ( $r s=.171, p=.019$ ). These findings could be aligned in parlance with the propositions of Miklousic et.al (2010), who noted that paranormal beliefs are closely associated with the traits of Neuroticism, Openness to experience, and Conscientiousness. Finally, as shown in the table, Spearman's rank order correlation analysis revealed that there was no significant relationship between Pre-cognition and any of the Personality traits. Thus, Hypothesis- 3 could be accepted in the case 7 combinations mentioned above (where significant correlation could be found), and has to be rejected otherwise.

The results of Stepwise Regression Analysis revealed that Conscientiousness is a significant predictor of Traditional religious beliefs $(\beta=.163, t=(190)=2.66, p=.025)$; Death $(\beta=$ $.123, t=(190)=1.70, p=0.13)$ Physical injury or illness $(\beta=.1 .22, t=(190)=1.70, p=$ $0.13)$, and Significant life experiences $(\beta=.133, t=(190)=1.83, p=.023)$ are significant predictors of the beliefs in Psi; and Death $((\beta=.193, t=(190)=2.71, p=.002)$, and Neuroticism $(\beta=.140, t=(190)=1.97, p=.002)$ are highly significant predictors of the beliefs in Witchcraft. It was found that Superstition could be predicted from Childhood traumatic experience of Death $(\beta=.186, t=(190)=2.54, p=.018)$, and Personality traits of Extraversion $(\beta=.024, t=(190)=.336, p=.018)$, Agreeableness $(\beta=-1.40, t=(190)=-$ $1.911, p=.018)$, and Neuroticism $(\beta=-0.32, t=(190)=-.431, p=.018)$. However, it has to be noted that the beliefs in Superstition was found to have a negative correlation with 
Extraversion and Agreeableness, thereby indicating the possibility of these two factors acting as negative predictors of the beliefs in Superstition. The analysis further revealed that Death $(\beta=.192, t=(190)=2.72, p=.001)$, and Openness to Experience $(\beta=.180, t=(190)=$ 2.556, $p=.001)$ are significant predictors of the beliefs in Spiritualism; and Death $(\beta=.207$, $t=(190)=2.891, p=.002)$, Significant life experiences $(\beta=.080, t=(190)=1.10, p=.002)$, and Openness to Experience $(\beta=.133, t=(190)=1.86, p=.002)$ are highly significant predictors of the beliefs in Extra-ordinary life forms. It was also found that Death is a highly significant predictor of the beliefs in Pre-cognition $(\beta=.168, t=(190)=2.34, p=.020)$.

\section{DISCUSSION}

Results of the study show the distribution of scores of early adults on Paranormal beliefs, Childhood trauma and Personality traits, and how each of these variables is related to each other. The results also indicate the distribution of paranormal beliefs across gender with special emphasize to each of the dimensions such as Traditional religious beliefs, Psi, Witchcraft, Superstition, Spiritualism, Extra-ordinary life forms, and Pre-cognition. Findings of the study are both congruent and incongruent to previous research findings and provide ample scope for further exploration.

The study found that the distribution of different dimensions of paranormal beliefs is not significant across categories of gender (males and females), except in the case of witchcraft. Even though females had higher mean rank scores than males in case of Traditional religious beliefs, beliefs in Psi, Superstition, and Pre-Cognition; the mean difference was found to be not significant. This finding is in similar lines with the proposition of Williams et al.(2007) that females (who are generally perceived to be more neurotic and emotional when compared to males) are more likely to have beliefs in the paranormal phenomena. Similarly, males were to have higher mean rank scores than females in case of the beliefs in Spiritualism and Extraordinary beliefs, but yet the mean difference was not statistically significant. More accurate findings appropriate to gender differences in holding onto paranormal beliefs could be derived out if the sample size employed for the study could be larger.

The study revealed that few dimensions of Childhood traumatic experiences and Paranormal beliefs are significantly correlated. As proposed by Irwin (1993), young adults who have undergone rigorous negative or traumatic experiences in their childhood exhibit a tendency to hold on to paranormal beliefs as a coping mechanism. This proposition derived out of the theoretical framework of 'Psychodynamic Functions Hypothesis' could be found appropriate to the context of the present study also. It was exposed that traumatic experience of Death or bereavement of a loved one prior to the age of 17 by individuals has been closely associated with their beliefs in Psi, Witchcraft, Superstition, Spiritualism, Extra-ordinary life forms, and Pre-cognition. Experience of any extreme Physical injury or illness was found to be significantly correlated to Psi. Occurrence of any other significant life event prior to the age of 17 was also seen to be associated with the beliefs in Psi and Extra-ordinary life forms. Early traumatic experiences of Divorce or separation between parents, Sexual abuse, and Violence Victimization were not found to be significantly associated with paranormal beliefs. 
Even though the distribution of scores of individuals on such domains was high, it was revealed that the relationship could not be considered as significant in the context of the present study. An expansion in the sample size and statistical rigor could probably yield better insights with regard to the relationship exiting between these domains of childhood trauma and paranormal beliefs. Interestingly, it was found that no dimension of childhood trauma has been closely associated with Traditional religious beliefs. This finding, which is contrary to the results of previous studies, in fact provides ample room for thought and investigation. Since Traditional religious beliefs are the commonest and robust form of paranormal beliefs prevailing among individuals, much focus should be given into this realm.

Since the belief in the paranormal is fairly prevalent in the contemporary society despite the overwhelming modernization of lives, previous studies have revealed that there exists a close alliance between Paranormal beliefs and Personality of the individuals. Results of the current study disclosed that all big-five domains of personality are correlated to at least one of the dimensions of paranormal beliefs. It was found that Conscientiousness and Traditional religious beliefs are having a positive correlation. Since individuals with Conscientiousness trait tend to conform to the social order too much, there is no wonder that such individuals might hold on to Traditional religious beliefs to the core. But no personality trait was revealed to be significantly correlated with the beliefs in Psi. Moving ahead, the trait of Neuroticism was found to be positively correlated with the beliefs in Witchcraft and Superstition, as claimed by previous studies. But Neuroticism was not found to be significantly correlated with any other dimension of paranormal beliefs. Hence, it could be presumed that the proposition of Williams et al. (2007) that individuals having neuroticism as their dominant trait of personality tend to have deeper beliefs in the paranormal phenomena than others, could not be accepted fully. Similarly, Openness to Experience was found to have a significant positive relationship with the beliefs in Spiritualism and Extra-ordinary life forms. Since individuals with the trait of openness are characterized by sensitivity and intellectual curiosity towards experiences, there is a high chance that they would rely on paranormal beliefs to introspect and fix up crises in life. Finally, the study also found that Agreeableness and Extraversion are having a significant negative correlation with the beliefs in Superstition. This finding which is contradictory to the previous findings that emphasized on a positive relationship between the variables should necessarily be subjected to further exploration. As mentioned earlier, the findings of the study may perhaps be much more refined if the sample size could be bigger.

In general, the study could identify certain significant predictors of paranormal beliefs ranging from Childhood experience of death, physical illness or injury, and significant life experiences; till Personality traits of Neuroticism, Conscientiousness, Extraversion, Agreeableness, and Openness to Experience. But unlike previous research findings, the relationship between certain dimensions of paranormal beliefs and the other two variables could not be established thoroughly. However, the age group and socio-cultural environment of the participants should also be taken into consideration before arriving at conclusions. Since the participants are early adults belonging to the age group 18-30 years, it should be 
noted that the home environment, education, job prospects, socio-economic status, and other situational factors could have been affecting their belief systems to a great extent. Accessibility of any other resources to cope up with the trauma induced by a negative event in the childhood also could have acted as negative catalyst in the formation of paranormal beliefs.

\section{CONCLUSION}

It was found that there is no significant difference in the distribution of paranormal beliefs across categories of gender, except in the case of beliefs in Witchcraft. The study revealed that the experience of Death or bereavement of a loved one in childhood has a significant correlation with the beliefs in Psi, Witchcraft, Superstition, Spiritualism, Extraordinary life forms, and Precognition. The experience of Physical illness or injury and other Significant life experiences were found to have a significant correlation with the beliefs in Psi. Beliefs in Extraordinary life forms were also found to have a positive relationship with the experience of Significant events in life prior to age 17. Finally, It was found that there exists a positive relationship between Conscientiousness and Traditional religious beliefs, Neuroticism and Witchcraft, Neuroticism and Superstition, Openness to Experience and Spiritualism; and Openness to Experience and beliefs in Extraordinary life forms. It was also found that there exists a significant negative relationship between the beliefs in Superstition and the traits of Extraversion and Agreeableness.

\section{LIMITATIONS OF THE STUDY}

Quantitative analysis would not suffice when it comes to the understanding of sensitive topics like belief systems and experiences. It would have been much better if some sort of qualitative analysis could be done to explore the influence of childhood trauma and personality traits on paranormal beliefs. Findings of the study are context-specific and may not be applied to other cultural settings. An understanding of the influence of childhood trauma and personality traits on paranormal beliefs below or above the age group 18-30 years is not possible, as the study is limited to this particular age group. Perhaps the situational and environmental factors could have impacted the results of the study.

\section{IMPLICATIONS OF THE STUDY}

India is one of the oldest countries where the beliefs in the unknown flourished and expanded amidst the people, irrespective of gender, status, or culture. But still, the volume of academic and experimental inquiries into this field is not sufficient in the regional context. More studies have to be initiated in the local milieu, in order to probe more into the Indian psyche from a psychological perspective. An exploration into the backdrop of beliefs in the paranormal phenomena among individuals hailing from the venerable culture of Kerala; anchored in heritage and religiosity, might be of assistance in this regard. Hence, this study could probably be reflected as an endeavor to unfurl mysteries hidden in the regional psyche. 


\section{Acknowledgments}

The author appreciates all those who participated and assisted in the study to facilitate the research process.

Conflict of Interests: The authors declare that there are no potential conflicts of interest with respect to the research, authorship, and/or publication of this article.

\section{REFERENCE}

Aarnio. K, \& Lindeman .M.(2006). Paranormal Beliefs: Their dimensionality and correlates. European Journal of Personality, 20, 585 - 602. doi : 10.1002/Per608

Huntley, C.,\& Peeters, T.(2005).Paranormal beliefs, religious beliefs and personality correlates(Thesis). Manchester Metropolitan University,UK

International Encyclopedia of Social Sciences. (1968). Retrieved from https://www.google.co.in/search?q=International+Encyclopedia+of+the+Social+Scie nces+\%7C+1968+COPYRIGHT+2008+Thomson+Gale.\&oq=International+Encyclop edia+of+the+Social+Sciences+\%7C+1968+COPYRIGHT+2008+Thomson+Gale.\&a qs=chrome..69i57.457j0j7\&sourceid=chrome\&ie=UTF-8

Irwin, H.J.(1993).Belief in the paranormal : A review of the empirical literature. Journal of the American Society for Psychical Ressearch. Retrieved from https:/www.researchgate.net/publication/239592095

Irwin, H. J. (1994). Childhood trauma and the origins of paranormal belief : a constructive application. Psychology representative, 74 (1), 107-11

John, O.P., \& Srivastava,S. (1999). The Big-Five trait taxonomy : History, measurement, and theoretical perspectives. In L.A Pervin \& O.P John (Eds), Handbook of personality : Theory and research (Vol.2, pp. 102-138). Newyork : Guildford press.

Mathew, V.G. (2005). Holigrative Psychology. Retrieved from http://www.psychology4all.com/vgm.htm

Mcdonald,A., \& Berkowski,M. (2014) Childhood trauma and the development of paranormal beliefs. Journal of Nerves and Mental Disabilities, 202 (4): 305-12. doi : 10.;1097/NMD 000000000000123

Miklousic,I., Mlacic,B.,\& Milas,G. (2010). Paranormal beliefs and personality traits in Croatia. doi : 10.555921.1.10

National Child Traumatic Stress Network. (2016). Retrieved from http://www.nctsn.org/trauma-types/early-childhood-trauma

Peltzer,K. (2002). Paranormal beliefs and personality traits among south African Black Students. Social behavior and Personality,30(4),pp.391-398.

Pennebaker ,J.W., \& Susman,J.R. (2013). Childhood trauma questionnaire. Measurement Instrument Database for the Social Science.Retrieved from www.midss.ie

Roe, C.A.,\& Bell, C. (2007) Paranormal belief, anxiety and perceived control over life events. In: Proceedings of the 50th Annual Parapsychological Association Conventiion. pp. 102-112. 
Rockville. (2014). Trauma-informed Care in Behavioural Health Services. US: Substance Abuse and Mental Health Services Administartion. Retrieved from http://www.ncbi.nlm.nih.gov/books/NBK207191/

Smith, C.L.,Johnson, J.L., \& Hathaway,W.(2009).Personality contributions to belief in Paranormal phenomena. Individual differences research,7(2),pp.85-96.

Tiwari,R.G. (n.d). What you Fear .... We chase !. Retrieved from www.indian paranormal society.com/publications.

Tobacyk ,J.J. (2004) A Revised paranormal belief scale. The International Journal of transpersonal studies23,94-98.

Williams,E., Francis, J.L., \& Robbins,M .(2007). Personality and paranormal belief : A study among adolescents. Retrieved from http: //dx.dot.org/10.1007/s11089-007-0094-x

How to cite this article: Prasad A \& Gopal B (2017). Influence of Childhood Trauma and Personality Traits on Paranormal Beliefs among Early-adults. International Journal of Indian Psychology, Vol. 5, (1), DIP: 18.01.114/20170501, DOI: 10.25215/0501.114 